Research Article

\title{
Role of piracetam on cognitive function in epilepsy and with antiepileptics in rats
}

\author{
Siddharth R. Chaudhari ${ }^{1}$, Priti P. Dhande ${ }^{2 *}$, Vijaya A. Pandit ${ }^{2}$
}

${ }^{1}$ Bristol-Myers Squibb India Pvt Ltd, Mumbai-13, Maharashtra, India

${ }^{2}$ Department of Pharmacology, Bharati Vidyapeeth (DU) Medical College, Pune- 43, Maharashtra, India

Received: 10 August 2013 Accepted: 18 August 2013

*Correspondence to:

Dr. Priti P. Dhande,

Email: ppdhande@yahoo.com

(C) 2013 Chaudhari SR et al. This is an open-access article distributed under the terms of the Creative Commons Attribution Non-Commercial License, which permits unrestricted non-commercial use, distribution, and reproduction in any medium, provided the original work is properly cited.

\begin{abstract}
Background: To study extent of cognitive impairment by epilepsy \& antiepileptic treatment and evaluate the role of piracetam on it.

Methods: 48 animals were divided into 6 groups: I-Control, II- Topiramate, IIITopiramate+Piracetam, IV-Valproate, V-Valproate+Piracetam, VI-Piracetam. Baseline cognitive functions were measured using Cook's pole climbing apparatus (CPCA) and Elevated plus maze (EPM). In CPCA, on completion of training, number of avoidances (NOA) out of 10 trials were noted while in EPM, transfer latency (TL) was measured. Kindling was induced by $30 \mathrm{mg} / \mathrm{kg}$ Pentylenetetrazol (PTZ), i.p. to all groups (except Group I) on alternate days till seizures developed. Groups were treated with respective drugs orally for 21 days and cognitive functions measured again.

Results: Significant decrease in NOA \& increase in TL was observed after PTZ kindling. Topiramate further significantly impaired NOA and TL whereas Valproate significantly reduced NOA in CPCA but increase in TL was not significant. Treatment with Piracetam significantly increased Topiramate, Valproate and PTZ kindling induced decrease in NOA as also significantly reduced Topiramate and PTZ kindling induced increase in TL.

Conclusion: Seizures are associated with cognitive impairment. Cognitive impairment caused by Sodium valproate differs from Topiramate. Piracetam, a known nootropic can be used in alleviating cognitive impairment associated with epilepsy \& chronic antiepileptic therapy.
\end{abstract}

Keywords: Cognition, Pentylenetetrazol, Kindling, Topiramate, Valproate, Piracetam

\section{INTRODUCTION}

Epilepsy refers to a disorder of brain function characterized by the periodic and unpredictable occurrence of seizures, attributable to abnormal synchronous neuronal activity. Such abnormal neuronal activity may have significant effect on the normal cognitive process and behavior of the affected individuals.

Epilepsy per se may induce or exacerbate an underlying cognitive impairment, but still a variety of factors contribute to such deficits, i.e., underlying neuropathology, etiology, seizure type, age of onset, severity, duration, seizure frequency, structural cerebral damage and psychosocial factors. ${ }^{1}$ Memory disturbances tend to be regarded as unavoidable sequelae of certain forms of epilepsy and only few experimental and clinical studies have been undertaken to mitigate these symptoms.
While the impact of epilepsy on normal brain activity is undesirable, the very drugs used to ameliorate seizures have shown to worsen learning and memory deficits often observed with the patients of epilepsy. By virtue of their mechanism of action, antiepileptic drugs (AEDs) decrease neuronal excitability and, therefore suppress epileptic form discharges. However, as they exert their effects indiscriminately, other neuronal networks that maintain normal neurocognitive functions may also become affected. AEDs may have an effect on specific cognitive functions but they most often have an impact on attention and central executive functions and they appear to impair the patient's quality of life more than seizure frequency. ${ }^{1,2}$

For example- Phenobarbitone, phenytoin, sodium valproate, carbamazepine are known for their cognitive side effects. Many newer antiepileptic drugs (e.g. 
gabapentin, lamotrigine, levetiracetam) have fewer adverse cognitive side effects than the older agents, yet studies are very limited and additional research is required to assess the cognitive side effect profile of the newer antiepileptic drugs.

Strategies, such as avoiding unnecessary polytherapy, using the lowest effective dose of a single AED, keeping drug levels within therapeutic ranges, and slow uptitration, have been associated with fewer cognitive side effects. Another interesting way to compensate cognitive dysfunction in epileptics could be the additive administration of 'memory enhancers' or nootropics like piracetam. Piracetam has been shown to improve learning and memory in a variety of experimental paradigms. ${ }^{3}$ Piracetam has been shown to alleviate phenytoin induced cognitive impairment without compromising its antiepileptic efficacy. ${ }^{4}$ But, yet studies have not been performed with other antiepileptic drugs and data is limited to comment on the exact role or utility of piracetam in epilepsy.

Therefore, a combination of antiepileptic drug with known nootropic agents appears to be a promising research area for desirable seizure control with minimal or no memory deficits and that is why this study was planned.

\section{METHODS}

The study was started after getting approval from Institutional Animal Ethics Committee of Bharati Vidyapeeth Deemed University Medical College, Pune (Approval Letter No. IAEC/ BVDUMC/ 06 / 2009-2010).

48 albino rats of either sex weighing 150-200gm were procured from the CPCSEA registered Central animal house of Bharati Vidyapeeth Medical College, Pune. Animals were housed with $12 \mathrm{hrs}$ day and night cycle and water was given ad libitum. Animals were screened before inclusion into the study by using Cook's pole climbing apparatus \& Elevated plus maze. Animals who demonstrated atleast one escape response out of ten trials in Cook's pole climbing apparatus and Transfer latency (TL) less than $60 \mathrm{sec}$ in Elevated plus maze were included in the study.

Animals were randomly assigned into six experimental groups and their baseline cognitive functions were assessed using both the above methods.

\section{Animal models to test cognitive function}

1. Cook's pole climbing apparatus ${ }^{5-7}$ : Animals were trained by turning on the buzzer signal of $50 \mathrm{~Hz}$ (conditioned stimulus) for $10 \mathrm{sec}$ followed by foot shock of $6 \mathrm{~mA}$ (unconditioned stimulus) for $10 \mathrm{sec}$. Maximum 20 trials/rat/day were given. Avoidance response was climbing the pole without shock (Reaction time <10sec) and Escape response was climbing the pole after shock
(Reaction time >10sec). When three consecutive avoidance responses were obtained, training was completed. $24 \mathrm{hrs}$ later a relearning trial (RT) composed of 10 trial session was carried out and number of avoidance responses were noted down as the baseline readings. Further readings of cognitive function (after PTZ-kindling and after completion of drug treatment), were taken by performing relearning trial to assess their long term memory of acquired conditioned responses.

2. Elevated plus maze $(E P M)^{8,9}$ : Experimental room was made sound proof $\&$ all the four arms of maze were equally illuminated. On day 1 of the experiment rat was placed at the end of an open arm with its head directed away from the central platform. Rat was allowed to move in one of the closed arm. If the animal did not enter in closed arm within $60 \mathrm{~s}$ it was gently pushed in the closed arm and to become acquainted with the maze, the rat was allowed to explore the maze for $10 \mathrm{~s}$ after reaching the closed arm and then returned to its home cage. On day 2 i.e. $24 \mathrm{hrs}$ after the exposure, again the rat was placed at the end of an open arm with its head directed away from the central platform \& the transfer latency (TL) was recorded. TL is the time taken by a rat to move from one of the open arm into one of the closed arm with all its four legs inside the closed arm. A time period of 300sec was taken as cut-off. Transfer latency $24 \mathrm{hrs}$ after exposure to EPM was recorded at every time point in experiment to assess short term memory of the rats.

\section{Pentylenetetrazol (PTZ) induced kindling ${ }^{4,10}$}

Kindling was induced by subconvulsive doses of $30 \mathrm{mg}$ PTZ/kg body weight of rat injected intraperitoneally (i.p.) once every $48 \mathrm{hrs}$ to all groups except group I. Animals in the group I received i.p. injection of distilled water $2 \mathrm{ml} / \mathrm{kg}$ body weight of rat. After each injection of PTZ the convulsive behavior was observed for $20 \mathrm{~min}$. The resultant seizures were classified as follows: Stage 0: no response; Stage 1: Hyperactivity, vibrissae twitching; Stage 2: Head nodding, head clonus and myoclonic jerk; Stage 3: Unilateral forelimb clonus; Stage 4: Rearing with bilateral forelimb clonus and Stage 5: Generalized tonic-clonic seizure (GTCS) with loss of writing reflex. Animals were considered kindled if they exhibited stage 5 of seizures on two consecutive trials. At this point of experiment, cognitive functions were again recorded in all the groups.

Groups: This was followed by drug treatment to respective groups orally for 21 days. Group I-Control (Distilled water), Group II-Topiramate $(2 \mathrm{mg} / 100 \mathrm{gm})$, Group III-Topiramate + Piracetam, Group IV-Valproate (100mg/100gm), Group V-Valproate + Piracetam, Group VI-Piracetam $(25 \mathrm{mg} / 100 \mathrm{gm})$. Third reading of cognitive functions was taken from $22^{\text {nd }}$ day of drug treatment.

Statistical analysis: Data was analyzed by using SPSS version 19 (Statistical Package for Social Sciences) software package. To compare the data at three different 
time point in each group, repeated measure ANOVA (Analysis of variance) was used. If the $p$ value was significant then the post hoc Bonferroni test was applied. $\mathrm{P}$-value $\leq 0.05$ was considered as significant.

\section{RESULTS}

The control group animals did not show any change in the cognitive function values throughout the experiment period.

As seen in Table 1 number of avoidance responses reduced significantly in PTZ kindled animals of group IIVI. In group II \& IV, drug treatment with Topiramate $(\mathrm{p}<0.0005) \&$ Valproate $(\mathrm{p}=0.001)$, further significantly decreased the number of avoidance responses in kindled rats. However in group III \& V, addition of Piracetam to Topiramate $(p=0.001) \&$ Valproate $(p=0.02)$ during the treatment period significantly improved the reduced conditioned avoidance response (CAR) values near to baseline. The animals in group VI also benefited from Piracetam in whom the impaired CAR caused by PTZ kindling significantly $(\mathrm{p}=0.001)$ reversed.

Table 2 shows that Transfer latency increased significantly after development of PTZ kindling in the experimental animals. 21 day Topiramate treatment in group II animals further significantly $(\mathrm{p}=0.008)$ increased the TL showing its deleterious effect on cognition in epileptic background. However in group IV, the impaired TL by kindling was not further altered by Valproate. Addition of Piracetam to Topiramate significantly $(\mathrm{p}=0.001)$ reduced the $\mathrm{TL}$ values near to baseline. In groups V \& VI, Piracetam significantly $(\mathrm{p}=0.004$ \& $0.008)$ reversed the impaired TL caused by PTZ kindling.

Table 1: Effect of PTZ kindling and drug treatment on conditioned avoidance response (CAR) in Cook's pole climbing apparatus in rats.

\begin{tabular}{|lllllll|}
\hline $\begin{array}{l}\text { No. of avoidance } \\
\text { responses at }\end{array}$ & $\begin{array}{l}\text { Group I } \\
\text { Control (DW) }\end{array}$ & $\begin{array}{l}\text { Group II } \\
\text { TPM }\end{array}$ & $\begin{array}{l}\text { Group III } \\
\text { TPM +PIM }\end{array}$ & $\begin{array}{l}\text { Group IV } \\
\text { VAL }\end{array}$ & $\begin{array}{l}\text { Group V } \\
\text { VAL +PIM }\end{array}$ & $\begin{array}{l}\text { Group VI } \\
\text { PIM }\end{array}$ \\
\hline Baseline & $9.25 \pm 0.16$ & $9.62 \pm 0.18$ & $9.25 \pm 0.31$ & $8.875 \pm 0.40$ & $8.75 \pm 0.36$ & $9.375 \pm 0.26$ \\
\hline After PTZ kindling & 9.0 & $6.12 \pm 0.52^{* *}$ & $6.75 \pm 0.31 * * *$ & $6.625 \pm 0.50 * * *$ & $6.0 \pm 0.60 * * *$ & $6.25 \pm 0.45 * * *$ \\
\hline $\begin{array}{l}\text { After 21 days drug } \\
\text { Treatment }\end{array}$ & $8.87 \pm 0.12$ & $3.0 \pm 0.46^{\$ \$ \$}$ & $9.0 \pm 0.19^{\$ \$ \$}$ & $4.0 \pm 0.42^{\$ \$}$ & $8.0 \pm 0.27^{\$}$ & $8.87 \pm 0.29^{\$ \$ \$}$ \\
\hline
\end{tabular}

DW- Distilled water, TPM- Topiramate, PIM- Piracetam, VAL- Valproate

Values are expressed as mean \pm SEM, $n=8$ animals in each group, Repeated Measure ANOVA followed by Bonferroni test for intragroup comparison

${ }^{* *} \mathrm{p} \leq 0.01,{ }^{* * *} \mathrm{p} \leq 0.001$ in comparison with the baseline readings

${ }^{\$} \mathrm{p} \leq 0.05,{ }^{\$} \$ \mathrm{p} \leq 0.001$ in comparison with post kindling values

Table 2: Effect of PTZ kindling and drug treatment on transfer latency in elevated plus maze in rats.

\begin{tabular}{|lllllll|}
\hline $\begin{array}{l}\text { Transfer } \\
\text { latency in } \\
\text { seconds at }\end{array}$ & $\begin{array}{l}\text { Group I } \\
\text { Control } \\
\text { (DW) }\end{array}$ & $\begin{array}{l}\text { Group II } \\
\text { TPM }\end{array}$ & $\begin{array}{l}\text { Group III } \\
\text { TPM + PIM }\end{array}$ & $\begin{array}{l}\text { Group IV } \\
\text { VAL }\end{array}$ & $\begin{array}{l}\text { Group V } \\
\text { VAL + PIM }\end{array}$ & $\begin{array}{l}\text { Group VI } \\
\text { PIM }\end{array}$ \\
\hline Baseline & $26.25 \pm 6.14$ & $48.0 \pm 6.03$ & $28.12 \pm 4.84$ & $22.0 \pm 4.69$ & $23.5 \pm 4.66$ & $24.0 \pm 3$ \\
\hline $\begin{array}{l}\text { After PTZ } \\
\text { kindling }\end{array}$ & $28.0 \pm 6.09$ & $108.87 \pm 19.02 *$ & $91.12 \pm 16.71 *$ & $104.0 \pm 14.75 * *$ & $71.87 \pm 13.02 * *$ & $85.87 \pm 17.61 *$ \\
\hline $\begin{array}{l}\text { After 21 days } \\
\text { drug Treatment }\end{array}$ & $27.375 \pm 5.32$ & $203.12 \pm 22.93^{\$ \$}$ & $44.62 \pm 11.36^{\$ \$ \$}$ & $109.12 \pm 12.74$ & $24.25 \pm 4.82^{\$ \$}$ & $33.25 \pm 8.73^{\$ \$}$ \\
\hline
\end{tabular}

DW- Distilled water, TPM- Topiramate, PIM- Piracetam, VAL- Valproate

Values are expressed as mean \pm SEM, $n=8$ animals in each group, Repeated Measure ANOVA followed by Bonferroni test for intragroup comparison

${ }^{*} \mathrm{p} \leq 0.05,{ }^{*} \mathrm{p} \leq 0.01$ in comparison with the baseline readings

${ }^{\$} \mathrm{p} \leq 0.01,{ }^{\$ \$} \mathrm{p} \leq 0.001$ in comparison with post kindling values 
As seen in Figure 1 there was difference in effect of 21 days treatment with Topiramate and Valproate on the transfer latency observed in Elevated plus maze in kindled rats. Topiramate significantly worsened the transfer latency in PTZ-kindled rats as contrast to Valproate where the difference is not evident.

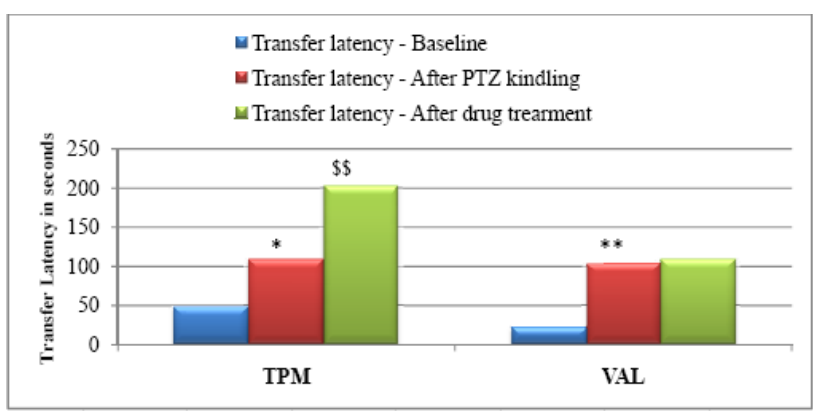

Figure 1: Comparison of change in transfer latency (TL) in elevated plus maze after Topiramate \& Valproate treatment in kindled rats.

TPM- Topiramate, VAL- Valproate

${ }^{*} \mathrm{p} \leq 0.05,{ }^{*} \mathrm{p} \leq 0.01$ in comparison with the baseline readings

${ }^{\$} \mathrm{p} \leq 0.01$ in comparison with post kindling values

\section{DISCUSSION}

One of the major problems encountered in patients of epilepsy is disturbance of learning and memory by epilepsy per se as well as the drugs used to treat the condition. Therefore the purpose of this study was to determine the potential of damage caused by epileptogenesis and antiepileptic drugs on memory parameters and to examine the effects of the nootropic drug Piracetam on learning and memory in rats.

\section{PTZ induced kindling and cognition}

Kindled animals in our study showed diminished performance in Cook's pole climbing apparatus and significantly increased transfer latency in the elevated plus maze which depicts impairment of memory. After PTZ-kindling, a significant neuronal cell loss has been found in all hippocampal structures except the subiculum. ${ }^{11}$ Ruethrich et $\mathrm{al}^{12}$ have demonstrated the development of a novel form of kindling-induced potentiation, in the dentate gyrus of PTZ kindled rats. PTZ, a GABA antagonist, leads to diminution of GABAergic inhibition and therefore would enhance glutamatergic transmission, which may be the cause of both seizure induction and intracellular metabolic changes leading to excitability enhancement and neuronal damage. ${ }^{4}$ Christine Rauca, et $\mathrm{al}^{13}$ show that a linkage exists between the PTZ- kindling and the increased formation of free radicals in brain. This fact may be an indication of the importance of free radicals generation with regard to the induction of processes mediating neuronal death during kindling. Elevation of free radicals in turn increase more basal release of excitatory amino acids. $^{14}$

This study has also demonstrated the protective effect of Piracetam on PTZ kindling induced cognition deficit. Piracetam produces an increase in the release and turnover of dopamine ${ }^{15}$, an increased release of acetylcholine in the hippocampus and corpus striatum ${ }^{16}$, an agonistic effect on serotonin (5-hydroxytryptamine; 5HT) receptors and an inhibiting mechanism on glutamate receptors. ${ }^{17}$ Pharmacodynamic investigations have demonstrated protective effects of piracetam against cerebral damage caused by hypoxia and ischaemia, and (in close connection with the biochemical and neurophysiological findings) benefits associated with memory and learning. ${ }^{18}$ Furthermore piracetam has also been shown to increase radical scavenge.Error! Bookmark not defined. These actions may explain the beneficial effects of piracetam on PTZ-kindling induced cognitive impairment.

\section{Topiramate and cognition}

Topiramate has a broad spectrum of application and generates the greatest concern over its potential negative neuropsychological effects. ${ }^{19,20}$ This study demonstrates that Topiramate $2 \mathrm{mg}$ / $\mathrm{kg}$ when given orally for 21 days to PTZ-kindled rats further worsens the performance of rats in both the cognition models. Agarwal NB et $\mathrm{al}^{21}$ have also found decreased step-down latency in passive avoidance response tests with topiramate $(10 \mathrm{mg} / \mathrm{kg}$, p.o.) in PTZ kindled mice in their experiment. In a multicenter, double-blind, randomized, prospective study conducted in adults with partial seizures where Lamotrigine or topiramate was introduced as an adjunctive therapy to carbamazepine or phenytoin, authors found that the frequencies of cognitive adverse events and of premature withdrawals related to cognitive decline were higher with topiramate than with lamotrigine. ${ }^{22}$ Pavone A and Cardile $\mathrm{V}^{22}$ have suggested that topiramate induces functional modifications and causes significant changes in NO production and enzymatic activity, ROS production in cortical rat astrocytes which in turn induces lipoperoxidation. This clarifies the neuronal toxicity caused by topiramate. It has also been found that repeated administration of topiramate significantly reduces the activity of glutamine synthetase (GS) which results in a diminished capacity for the brain to metabolize, and thereby detoxify glutamate. Interestingly, those drugs with an inhibitory action on the enzyme exhibit CNSrelated toxicity, such as ataxia, sedation, dizziness and cognitive impairment. ${ }^{23}$

\section{Valproate and cognition}

Though the cognitive effects of Valproate are mild to moderate, they have been reported in animal studies ${ }^{24}$ and clinical data. ${ }^{19,25}$ Valproate is frequently used as the "gold standard" 26,27 to examine the cognitive effects of newer 
AEDs. The present study signifies that valproate affects only long term retention of conditioned response and has no effect on short term retention of transfer latency in kindled rats. A study by Umka $\mathrm{J}$ et $\mathrm{al}^{28}$ shows that valproate can cause cognitive impairments which are associated with changes in hippocampal neurogenesis and neurotrophin levels. These changes may indicate several possible mechanisms of valproate action including reducing cell proliferation in the subgranular zone of the dentate gyrus within the hippocampus, altering neuronal maturation and synaptic plasticity.

\section{Piracetam with topiramate and valproate}

Piracetam given orally along with topiramate significantly improved the performance of kindled animals in both the cognition models while it may have prevented the negative effect of valproate on conditioned response in the Cook's pole climbing test. From the observations it can be said that piracetam may have not only prevented the cognitive deterioration caused by these drugs but also enhanced the cognitive performance of the study animals because of its neuroprotective and nootropic action.

Sansone $M$ and Oliverio $\mathrm{A}^{29}$ have demonstrated facilitation of active avoidance acquisition by piracetam in the shuttle-box test in mice. Shahid $\mathrm{M}$ et $\mathrm{al}^{5}$ conclude from their study on effect of the combined treatment of phenytoin and piracetam on seizure control, cognitive and motor functions in mice, that piracetam when coadministered with therapeutic doses of phenytoin it significantly alleviated the adverse effects of phenytoin on cognitive function without compromising its antiepileptic efficacy.

Mechanisms for positive effect of piracetam on cognition found in our study when given along with topiramate or valproate in the kindled rats might be enhancement of oxidative glycolysis, effect on cholinergic system ${ }^{5}$, inhibitory action on glutamate receptors ${ }^{18}$ and improvement of mitochondrial dysfunction associated with oxidative stress $^{30}$ which have been reported in studies to find out its exact role in central nervous system.

\section{CONCLUSION}

Pentylenetetrazol induced kindling significantly showed impaired cognition in both the memory paradigms which was further worsened by topiramate in kindled rats. Valproate was seen to affect only the long term conditioned response in kindled rats while the short term latency in the elevated plus maze test was not significantly affected. Piracetam appears to be counteracting the cognitive deficit in epilepsy as well as due to the study antiepileptic drugs. Therefore it is likely that piracetam has a beneficial role in epilepsy and in combination with antiepileptics by improving the cognitive profile in both these situations.
Funding: None

Conflict of interest: None declared

Ethical approval: The study was approved by the Institutional Animal Ethics Committee of Bharati Vidyapeeth Deemed University Medical College, Pune (Approval Letter No. IAEC/ BVDUMC/ 06 / 2009-2010)

\section{REFERENCES}

1. Motamedi G, Meador K. Epilepsy and cognition. Epilepsy Behav 2003 Oct;4(2):25-38.

2. Hermann B, Seidenberg M. Epilepsy and cognition. Epilepsy Curr 2007 Jan-Feb;7(1):1-6.

3. Nicholson CD. Pharmacology of nootropics and metabolically active compounds in relation to their use in dementia. Psychopharmacology 1990; 101(2): 147-59.

4. Shahid M, Pillai KK, Vohora D. Reversal of phenytoin-induced impairment of spontaneous alternation by piracetam in mice: Involvement of cholinergic system. Indian J Pharmacol 2004;36: 20-4.

5. Cook L, Weidley E. Behavioral effects of some psychopharmacological agents. Ann N Y Acad Sci 1957;66(3):740-52.

6. Soman I, Mengi SA, Kasture SB. Effect of leaves of Butea frondosa on stress, anxiety, and cognition in rats. Pharmacol Biochem Behav 2004;79(1):11-6.

7. Saito H, Tsuchiya M, Naka S, Takagi K. Effects of Panax Ginseng root on conditioned avoidance response in rats. Jpn J Pharmacol 1977;27(4):50916.

8. Walf AA, Frye CA. The use of the elevated plus maze as an assay of anxiety-related behavior in rodents. Nat Protoc 2007;2(2):322-8.

9. Itoh J, Nabeshima T, Kameyama T. Utility of an elevated plus-maze for the evaluation of memory in mice: effects of nootropics, scopolamine and electroconvulsive shock. Psychopharmacology 1990;101(1):27-33.

10. Mehla J, Reeta KH, Gupta P, Gupta YK. Protective effect of curcumin against seizures and cognitive impairment in a Pentylenetetrazol-kindled epileptic rat model. Life Sci 2010 Nov 20;87(19-22):596-603.

11. Pohle W, Becker A, Grecksch G, Juhre A, Willenberg A. Piracetam prevents Pentylenetetrazol kindling-induced neuronal loss and learning deficits. Seizure 1997;6(6):467-74.

12. Ruethrich H, Grecksch G, Becker A, Krug M. Potentiation effects in the dentate gyrus of Pentylenetetrazol-kindled rats. Physiol Behav 1996; 60(2):455-62.

13. Rauca C, Zerbe R, Jantze H. Formation of free hydroxyl radicals after Pentylenetetrazol-induced seizure and kindling. Brain Res 1999;847(2):347-51.

14. Gilman SC, Bonner MJ, Pellmar TC. Free radicals enhance basal release of $\mathrm{D}-[3 \mathrm{H}]$ aspartate from cerebral cortical synaptosomes. J Neurochem 1994; 62(5):1757-63. 
15. Nyback H, Wiesel FA, Skett P. Effects of piracetam on brain monoamine metabolism and serum prolactin levels in the rat. Psychopharmacology 1979;61(3):235-8.

16. Wurtman RJ, Magil SG, Reinstein DK. Piracetam diminishes hippocampal acetylcholine levels in rats. Life Sci 1981; 28(10):1091-3.

17. Bering B, Müller WE. Interaction of piracetam with several neurotransmitter receptors in the central nervous system. Relative specificity for $3 \mathrm{H}-$ glutamate sites. Arzneimittelforschung 1985;35(2): 1350-2.

18. Hitzenberger G, Rameis H, Manigley C. Pharmacological Properties of Piracetam. Rationale for Use in Stroke Patients. CNS Drugs 1998; 9 (Suppl. 1):19-27.

19. Carreño M, Donaire A, Sánchez-Carpintero R. Cognitive disorders associated with epilepsy: diagnosis and treatment. Neurologist 2008;14 (6 Suppl 1):S26-34.

20. Blum D, Meador K, Biton V, Fakhoury T, Shneker $\mathrm{B}$, Chung S, et al. Cognitive effects of lamotrigine compared with topiramate in patients with epilepsy. Neurology 2006;67(3):400-6.

21. Agarwal NB, Agarwal NK, Mediratta PK, Sharma KK. Effect of lamotrigine, oxcarbazepine and topiramate on cognitive functions and oxidative stress in PTZ-kindled mice. Seizure 2011; 20(3): 257-62.

22. Pavone A, Cardile V. An in vitro study of new antiepileptic drugs and astrocytes. Epilepsia 2003;44 (Suppl 10):34-9.

23. Fraser CM, Sills GJ, Forrest G, Thompson GG, Brodie MJ. Effects of anti-epileptic drugs on glutamine synthetase activity in mouse brain. $\mathrm{Br} \mathrm{J}$ Pharmacol 1999;126(7):1634-8.

24. Higgins GA, Breysse N, Undzys E, Derksen DR, Jeffrey M, Scott BW, et al. Comparative study of five antiepileptic drugs on a translational cognitive measure in the rat: relationship to antiepileptic property. Psychopharmacology 2010;207(4):513-27.

25. Kwan P, Brodie MJ. Neuropsychological effects of epilepsy and antiepileptic drugs. Lancet 2001 Jan; 357: 216-22.

26. Aldenkamp AP, Baker G, Mulder OG, Chadwick D, Cooper P, Doelman J, et al. A multicenter, randomized clinical study to evaluate the effect on cognitive function of topiramate compared with valproate as add-on therapy to carbamazepine in patients with partial-onset seizures. Epilepsia 2000; 41(9):1167-78

27. Meador KJ, Loring DW, Hulihan JF, Kamin M, Karim R. Differential cognitive and behavioral effects of topiramate and valproate. Neurology 2003; 60(9):1483-8.

28. Umka J, Mustafa S, ElBeltagy M, Thorpe A, Latif L, Bennett $G$, et al. Valproic acid reduces spatial working memory and cell proliferation in the hippocampus. Neuroscience 2010; 166(1):15-22.

29. Sansone M, Oliverio A. Avoidance facilitation by nootropics. Prog Neuropsychopharmacol Biol Psychiatry 1989;13:S89-97.

30. Keil U, Scherping I, Hauptmann S, Schuessel K, Eckert A, Müller WE. Piracetam improves mitochondrial dysfunction following oxidative stress. Br J Pharmacol 2006;147(2):199-208.

doi: 10.5455/2319-2003.ijbcp20131022

Cite this article as: Chaudhari SR, Dhande PP, Pandit VA. Role of piracetam on cognitive function in epilepsy and with antiepileptics in rats. Int J Basic Clin Pharmacol 2013;2:634-9. 